\title{
Post-transcriptional gene silencing in plants: a double-edged sword
}

\author{
Xinyan Zhang ${ }^{1,3}$, Ying Zhu ${ }^{1}$, Huihui $\mathrm{Wu}^{1} \&$ Hongwei Guo ${ }^{1,2^{*}}$ \\ ${ }^{1}$ State Key Laboratory of Protein and Plant Gene Research, College of Life Sciences, Peking University, Beijing 100871, China; \\ ${ }^{2}$ Peking-Tsinghua Center for Life Sciences, Peking University, Beijing 100871, China; \\ ${ }^{3}$ Present address: Department of Botany and Plant Pathology, Purdue University, West Lafayette IN 47907, USA
}

Received August 18, 2015; accepted September 8, 2015; published online December 29, 2015

\begin{abstract}
In plants, post-transcriptional gene silencing (PTGS) protects the genome from foreign genes and restricts the expression of certain endogenous genes for proper development. Here, we review the recent progress about how the unwanted PTGS is avoided in plants. As a decision-making step of PTGS, aberrant transcripts from most endogenous coding genes are strictly sorted to the bidirectional RNA decay pathways in cytoplasm but not to the short interference RNA (siRNA)-mediated PTGS, with the exception of a few development-relevant endogenous siRNA-producing genes. We also discuss a finely balanced PTGS threshold model that plants fully take advantage of the power of PTGS without self-harm.
\end{abstract}

post-transcriptional gene silencing, short interference RNA, coding-transcript-derived siRNA, RNA decay

Citation: $\quad$ Zhang, X., Zhu, Y., Wu, H., and Guo, H. (2016). Post-transcriptional gene silencing in plants: a double-edged sword. Sci China Life Sci 59 , $271-276$. doi: $10.1007 / \mathrm{s} 11427-015-4972-7$

\section{INTRODUCTION}

Coordinated gene expression and silencing allows for the establishment of the overall transcriptome in eukaryotic cells. Gene silencing occurs either transcriptionally or post-transcriptionally, with post-transcriptional gene silencing (PTGS) commonly serving as an RNA-based immune mechanism in eukaryotes, protecting against virus and foreign gene invasion. Meanwhile, the PTGS pathway is embedded in cellular regulatory networks, playing key roles in growth and development (Cogoni and Macino, 2000; Ghildiyal and Zamore, 2009). In plants, transgene PTGS, also known as cosuppression, was first described in transgenic plants in which the expression of both transgenes and their cognate endogenous genes was disrupted (Napoli et al., 1990; Van der Krol et al., 1990). Early discoveries such as RNA-based viral resistance in plants (Lindbo et al., 1993), the quelling effect in fungi (Cogoni et al., 1994), and

*Corresponding author (email: hongweig@pku.edu.cn)
RNA interference in animals (Fire et al., 1998) share similar underlying PTGS mechanisms. In contrast to the highly expressed transgenes and invading viral genes that frequently undergo PTGS, the expression of most endogenous genes does not trigger PTGS, with the exception of a few endogenous siRNA production genes (Chen, 2009). These observations raise questions about how PTGS is regulated in cells, and how most endogenous coding transcripts avoid PTGS. Here, we review our current understanding of the decision-making mechanisms involved in the sorting of cellular transcripts for gene expression or PTGS, and emphasize the role of cytoplasmic RNA decay pathways in the maintenance of a finely balanced PTGS threshold, whereby transcripts from foreign genes are channeled into the PTGS pathway, while endogenous coding genes are protected from silencing.

\section{TRANSGENE PTGS AND ENDOGENOUS PTGS}

PTGS is trigged by cellular double-stranded RNAs (dsR- 
NAs) which are recognized and processed into 20-22 nucleotide (nt) RNA duplexes by Dicer family proteins (Hutvagner, 2001; Xie et al., 2004). One strand of the small RNAs, such as small interfering RNA (siRNA) duplexes processed by DCL2 (Dicer-like 2) and DCL4 (Borsani et al., 2005; Chen, 2005; Hamilton and Baulcombe, 1999) and microRNA (miRNA) duplexes processed by DCL1 (Chen, 2005), can be loaded into the Argonaute (AGO)containing RNA-induced silencing complex (RISC), resulting in mRNA cleavage or translational inhibition (Fabian et al., 2010; Sontheimer, 2005). In plants, worms and some fungi, the primary PTGS effect can be amplified by triggering an additional round of siRNA production from the target transcripts in a manner requiring the involvement of RNA-dependent RNA polymerases (RdRPs). This process is referred to as secondary siRNA biogenesis and follows either primary miRNA- or siRNA-directed mRNA cleavages; it may also occur following both processes (Sijen et al., 2001; Vaistij et al., 2002; Voinnet et al., 1998). Noticeably, a subset of the secondary siRNAs, known as epigenetically activated siRNAs (easiRNAs), is engaged in the defense of plant genomes against derepressed transposons (Creasey et al., 2014).

Eukaryotic PTGS is regulated by multiple exogenous and endogenous factors. For instance, various plant viruses encode PTGS suppressor proteins to counteract the host PTGS systems and thus carry out a successful invasion, by either attenuating plant PTGS core effectors such as RDR1, RDR6, and SGS3, or efficiently inhibiting dsRNA processing and RISC assembly (Incarbone and Dunoyer, 2013; Pumplin and Voinnet, 2013). In addition, numerous key components involved in mRNA quality control, processing, and degradation have been demonstrated to be repressors of transgene PTGS (Gazzani et al., 2004; Gy et al., 2007; Martinez de Alba et al., 2015; Moreno et al., 2013; Thran et al., 2012).

To identify repressors of transgene PTGS, several research groups have performed genetic screens based on the form of transgene cosuppression wherein the mutants undergoing transgene PTGS phenocopy the loss-of-function mutants of the cognate endogenous genes. These screens have yielded descriptions of several forms of cytoplasmic RNA decay machinery, such as the Arabidopsis cytoplasmic 5'-3' exoribonuclease, EIN5/XRN4, and the 3'-5' SKIexosome complex (Gazzani et al., 2004; Zhang et al., 2015). In eukaryotes, decapped or depolyadenylated transcripts undergo efficient degradation in the cytoplasm. EIN5/ XRN4 is known as the only cytoplasm-localized 5'-3'exoribonuclease in Arabidopsis, and has been identified as a repressor of virally induced vasiRNA biogenesis from many host genome loci (Cao et al., 2014). The cytoplasmic SKI complex composed of SKI2, SKI3, and SKI8 has been well characterized, both structurally and functionally. It is a part of the cytoplasmic $3^{\prime}-5^{\prime}$ RNA decay pathway, threading RNAs into the yeast exosome (Halbach et al., 2013). Previ- ously, the functional significance of EIN5 and the nature of the SKI-exosome machineries in plants were unclear, for two reasons: (i) the cytoplasmic RNA decay pathways are evolutionarily conserved in eukaryotes, but neither are essential to plant development, a conclusion based on the normal development of the ski2 and ski3 single mutants and the mild phenotypic defects manifested in the null ein $5 \mathrm{mu}-$ tant (Gregory et al., 2008; Olmedo et al., 2006; Potuschak et al., 2006; Zhang et al., 2015). Few lines of evidence convey the significance of the role played by the SKI-exosome in the regulation of endogenous PTGS; however, CER7, a core subunit of the exosome, has recently been demonstrated to repress a class of tasiRNA biosynthesis, some types of which affect wax deposition in inflorescence stem development (Lam et al., 2015). In the ein5 mutant, siRNA production has been observed affect $~ 130$ endogenous genes that normally do not produce siRNAs (Gregory et al., 2008), suggesting that EIN5 might play a role in the repression of endogenous PTGS. However, the functional consequences of these siRNAs are not yet clear. (ii) If the major function of RNA decay is to repress the silencing of foreign genes, a mechanism underlying RNA-based plant immunity, these machineries should have been selected against in evolution. Our recent study reveals that concomitant loss of cytoplasmic 5'-3' and $3^{\prime}-5^{\prime}$ RNA decay pathways results in severe developmental defects accompanied by abundant endogenous siRNA biogenesis (discussed in the following section) and drastic transcriptome perturbation, both of which are substantially suppressed by PTGS mutants, indicating that the bidirectional RNA decay pathways repress endogenous PTGS (Zhang et al., 2015). Moreover, aversion of the PTGS of endogenous genes seems to be a major cellular function of cytoplasmic RNA decay, because both RNA decay pathways are dispensable when the PTGS pathway is inactivated. Therefore, the bidirectional RNA decay pathways in cytoplasm form an anti-PTGS module in order to ensure proper endogenous gene expression (Zhang et al., 2015).

It is hypothesized that the overexpression of transgenes may override the endogenous RNA processing machineries, leading to the production of abnormal mRNAs, which are then subject to the RDR6-mediated gene silencing pathway (Zhang et al., 2015). In accordance with this model, high levels of transgene expression are usually associated with high frequencies of transgene-induced co-suppression in plants (Lindbo et al., 1993; Schubert et al., 2004; Vaucheret et al., 1998). While cytoplasmic RNA decay suppresses the PTGS of both endogenous and foreign genes, there is a discrepancy between the two. In fact, either the $5^{\prime}-3^{\prime}$ or $3^{\prime}-5^{\prime}$ pathway is sufficient for the suppression of endogenous detrimental PTGS, as evidenced by the normal development of single ein5 and ski2 single mutants, whereas both pathways are necessary for the prevention of transgene PTGS, as the loss of either ein 5 or ski2 results in the cosuppression of transgenes (Zhang et al., 2015). A threshold model has been proposed, in which excessive aberrant tran- 
scripts of transgene expression can occasionally trigger PTGS by breaking through the bidirectional RNA decay, while on the other hand the high threshold for endogenous PTGS set up by RNA decay is too high for the phenomenon to occur frequently.

\section{A NOVEL CLASS OF ENDOGENOUS SECONDARY siRNAS (ct-siRNAS) IN PTGS}

The production of siRNAs triggers PTGS. In Arabidopsis mutants defective in bidirectional cytoplasmic RNA decay, a class of novel endogenous siRNAs, coding-transcriptderived siRNAs (ct-siRNAs), has been identified (Zhang et al., 2015). Those ct-siRNAs exhibit the following characteristics: (i) 21-22 nt in length; (ii) derived from coding transcripts; (iii) RDR6-dependent in biogenesis; and (iv) partially AGO1-dependent in function. Interestingly, this class of endogenous siRNAs is hardly detected in wild-type plants, indicating that the biosynthesis of ct-siRNAs from coding genes is prevented through the efficient elimination of aberrant transcripts by cytoplasmic RNA decay.

Being a novel class of secondary siRNAs, ct-siRNAs differ in both biogenesis and function from trans-acting siRNAs (tasiRNAs), a class of well-characterized secondary siRNAs derived from some non-coding transcripts (Allen et al., 2005; Axtell et al., 2006; Montgomery et al., 2008; Vazquez et al., 2004). Firstly, the biogenesis of ct-siRNAs is repressed by cytoplasmic RNA decay, while that of tasiRNAs bypasses RNA decay. Secondly, while miRNA-mediated mRNA cleavage triggers the biogenesis of both ct-siRNAs (in the absence of RNA decay) and tasiRNAs, ct-siRNAs are preferentially generated from 5' miRNA cleavage fragments, seldom manifesting unique starting positions at the cleavage sites. It is speculated that either certain $3^{\prime}$ end processing of $5^{\prime}$ cleavage products occurs prior to dsRNA biogenesis (Ren et al., 2014), or RDR6 utilizes various entry-sites at the $3^{\prime}$ end of $5^{\prime}$ miRNA cleavage products when catalyzing dsRNA formation. Conversely, tasiRNAs are mainly produced from $3^{\prime}$ intermediates with unique starting position, and mostly exhibit 21 -nt periodicity because of consecutive processing by DCL4. The function of tasiRNAs relies on the precise phasing of mRNA processing, which efficiently generates in-phase trans-acting siRNAs; otherwise, nucleotide shifts may disrupt tasiRNA targeting and cause the tasiRNA module to function inefficiently. This could be one explanation for why the $3^{\prime}$ RNA intermediates produced during miRNA cleavage are evolutionarily favored as tasiRNA-producing templates (Allen et al., 2005; Axtell et al., 2006). Lastly, in term of function, ct-siRNAs interfere with endogenous gene expression, which is primarily detrimental to plant development; meanwhile, tasiRNAs are integrated into the normal developmental network, and are evolutionarily selected for.

In particular, ct-siRNAs have been demonstrated to be deleterious to the plant miRNA regulatory network. The regulation of endogenous gene expression by miRNAs is usually associated with mRNA cleavages and the production of aberrant transcripts (Chen, 2005). The risks of these miRNA-directed aberrant mRNAs have been demonstrated via genetic analysis using mutants exhibiting defective bidirectional cytoplasmic RNA decay (Zhang et al., 2015). Among the ct-siRNA-generating loci, miRNA targets are overrepresented and many exhibit compromised gene expression due to the dysfunction of bidirectional RNA decay. Hence, cytoplasmic RNA decay likely prevents a sustained shutdown of gene expression following primary miRNA targeting, in order to preserve a basal expression level. In addition, the recovery of mRNA abundance can be achieved promptly upon the withdrawal of miRNAs. Nevertheless, it is hypothesized that some miRNA actions require ct-siRNA biogenesis to fully silence their target transcripts when necessary or transitively silence other related transcripts in order to regulate a broader range of genes. Examples of these miRNA-secondary siRNA modules have been found to regulate large conserved gene families such as the NB-LRR (nucleotide-binding and leucine-rich repeat) proteins, MYB transcription factors, PPR (pentatricopeptide repeat) proteins, and F-box families (Xia et al., 2012; Xia et al., 2013; Xia et al., 2015; Zhai et al., 2011).

\section{MULTIPLE SAFEGUARD MECHANISMS TO AVERT ADVERSE ENDOGENOUS PTGS}

In addition to the RNA decay pathways, the interaction of diversified DCL family proteins serves as one more layer of PTGS regulation. DCL proteins are key enzymes in the processing of dsRNAs. There are four DCL proteins in $\mathrm{Ar}$ abidopsis, among which DCL4 and DCL2 are required for the production of 21- and 22-nt siRNAs, respectively. DCL1 is specifically involved in miRNA production, while DCL3 is involved in 24-nt heterochromatic siRNA biogenesis. Interestingly, dcll $d c l 4$ and $d c l l ~ d c l 3 d c l 4$ mutants manifest a growth arrest and frequent seedling lethality, whereas $d c l l d c l 2 d c l 4$ and $d c l l d c l 2 d c l 3 d c l 4$ mutants are viable (Bouche et al., 2006). It is speculated that the growth defects in $d c l l d c l 4$ and $d c l l d c l 3 d c l 4$ are due to the overproduction of 22 nt siRNA by DCL2, which results in explosive secondary siRNA biogenesis, affecting the transcriptome (Chen et al., 2010; Cuperus et al., 2010; Gasciolli et al., 2005; Xie et al., 2005). Our recent studies have further clarified both cooperative and antagonistic relationships between DCL4 and DCL2 in siRNA biogenesis (Zhang et al., 2015). When the RNA decay mutants (ein5 or ski2) are combined with the dcl4 mutant, the double mutants manifests pleiotropic developmental defects, which are rescued by further loss of DCL2. The genetic interactions between DCL proteins and the cytoplasmic RNA decay pathway emphasize a key function of DCL4, which in compet- 


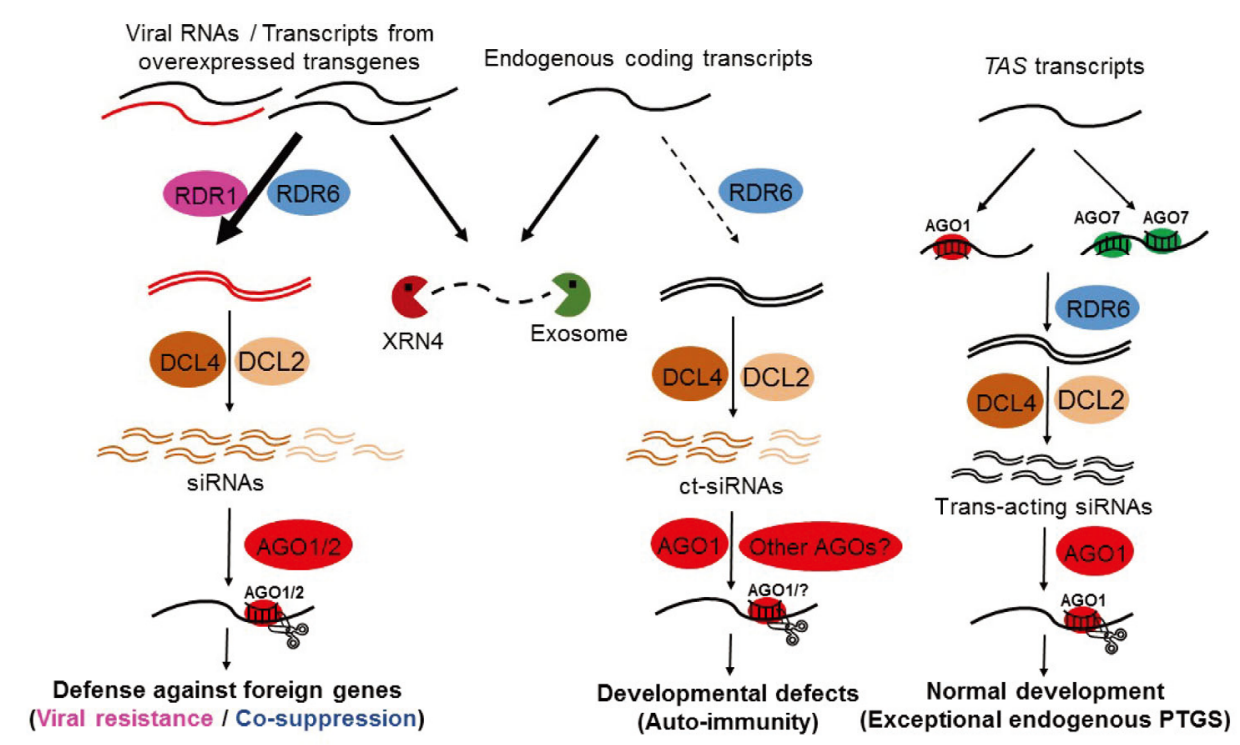

Figure 1 Biogenesis of siRNAs that mediate plant immunity and development. Aberrant mRNAs are substrates of the 5'-3' (EIN5) and 3'-5' (SKI-Exosome) mRNA processing machineries in cytoplasm. Both $5^{\prime}-3^{\prime}$ and $3^{\prime}-5^{\prime}$ RNA decay pathways are crucial to the repression of transgene-induced PTGS. In the absence of both mRNA processing pathways, aberrant mRNAs accumulate and are subsequently subjected to RDR6-mediated ct-siRNA production; therefore, the cytoplasmic mRNA decay pathways function as double safeguards preventing the PTGS of endogenous coding genes and repressing the transition from a normal developmental progress to a PTGS-dependent self-destructive process like auto-immunity. Exceptional endogenous PTGS in the wild-type plants is mediated by the trans-acting siRNAs, which are development-relevant and produced in a manner that bypasses RNA decay in the cytoplasm.

ing with DCL2, helps ease the PTGS pressure on endogenous aberrant transcripts in the absence of either $5^{\prime}-3^{\prime}$ or $3^{\prime}-5$ ' RNA decay pathways. Altogether, the DCL4-mediated 21-nt siRNA pathway functions as a PTGS decoy and serves as an additional safeguard, in addition to the bidirectional RNA decay in the cytoplasm, which acts against ct-siRNA-mediated endogenous PTGS (Zhang et al., 2015).

Averting the PTGS of endogenous genes seems to be the most important function of bidirectional cytoplasmic mRNA decay. Based on genetic evidence, it is likely that cells might be capable of tolerating high levels of aberrant mRNAs via certain unknown mechanisms, and that cytoplasmic aberrant mRNAs have few harmful effects other than the triggering of secondary siRNA biosynthesis in plants. Alternatively, there is still a possibility that cytosolic aberrant mRNA lingers prior to being actively degraded by other cellular components. For example, ISE2, a SKI2 homolog in the cytosol, seems to be important for plant development, as ise 2 mutants are seedling lethal and exhibit defects in the functionality of plasmodesmata (Kobayashi et al., 2007). Interestingly, it has also been suggested that ISE2 plays a role in transgene silencing, although it seems to function as a PTGS enhancer rather than a repressor like SKI2 (Kobayashi et al., 2007).

It has been recently reported that the decapping complex, active in mRNA quality control, is crucial to plant development, partially because of its function in preventing endogenous PTGS. This is evidenced by the fact that RDR6 partially restores the developmental defects present in $d c p 2$ and $v c s$ mutants and prevents accumulation of a class of
RNA-quality-control siRNAs (rqc-siRNAs) (Martinez de Alba et al., 2015). This independent evidence also emphasizes the crucial roles played by RNA processing and degradation in the repression of endogenous PTGS.

\section{CONCLUSIONS AND PERSPECTIVES}

To produce an on-or-off PTGS response, plants employ a module composed of (i) aberrant RNA triggers that initiate the PTGS, (ii) an RNA-decay-based brake system that efficiently eliminates triggers and sets up a PTGS threshold, (iii) and positive feedback via secondary siRNA amplification that maintains the PTGS when turned on. This bistable module is critical for cell immunity that unambiguously recognizes self and non-self transcripts in the cytoplasm, and sorts them into either expression or silencing pathways.

According to studies conducted in plants, the crucial roles of bidirectional mRNA decay pathways can be inferred to be involved in the repression of the PTGS in some fungi and animals, which are also equipped with RDRPs for the amplification of PTGS (Ghildiyal and Zamore, 2009). Nonetheless, the possible cooperation of two mRNA processing pathways and its context-dependent biological significance require further studies to be carried out in various organisms. For example, the yeast xrn1 ski2 double mutant is also synthetic lethal (Johnson and Kolodner, 1995), implying the mRNA decay pathways are also indispensable to organisms without siRNA amplification, including budding yeast, fly, mouse, and humans. However, to our knowledge, 
the suppressor screening for mutants that rescue the lethality of the yeast xrn 1 ski2 double mutant resulted in the isolation of only one mutant, ratl, in which the detained RAT1 proteins in the cytosol functionally substitute XRN1 (Johnson, 1997). Further studies are required to elucidate the functions of the cytosolic mRNA decay pathways in various organisms.

Altogether, gene expression is a risk-taking process in the presence of cellular gene-silencing machineries, and the bidirectional cytoplasmic RNA decay plays a key role in guarding normal plant development from PTGS-elicited self-attack, a process somewhat in analogous to auto-immunity in animals (Marmont, 2000). The maintenance of a certain threshold for PTGS is a trade-off, because plants without PTGS will be susceptible to viral infection and are impotent for the control of transgene expression, whereas plants possessing a low PTGS threshold have compromised robustness of endogenous gene expression. The high risk of overrunning ct-siRNA biogenesis provides one explanation for why RNA-based immunity gradually faded away and switched to protein-based immunity in evolution (Li et al., 2013; Maillard et al., 2013).

Compliance and ethics The author(s) declare that they have no conflict of interest.

Acknowledgements We thank the members of the Guo lab for discussing our work with us. This work was supported by the National Basic Research Program of China (2012CB910902) and the National Natural Science Foundation of China $(91217305,91017010)$ to H.G.

Allen, E., Xie, Z., Gustafson, A.M., and Carrington, J.C. (2005). microRNA-directed phasing during trans-acting siRNA biogenesis in plants. Cell 121, 207-221.

Axtell, M.J., Jan, C., Rajagopalan, R., and Bartel, D.P. (2006). A two-hit trigger for siRNA biogenesis in plants. Cell 127, 565-577.

Borsani, O., Zhu, J., Verslues, P.E., Sunkar, R., and Zhu, J.K. (2005). Endogenous siRNAs derived from a pair of natural cis-antisense transcripts regulate salt tolerance in Arabidopsis. Cell 123, 1279-1291.

Bouche, N., Lauressergues, D., Gasciolli, V., and Vaucheret, H. (2006). An antagonistic function for Arabidopsis DCL2 in development and a new function for DCL4 in generating viral siRNAs. EMBO J. 25, 3347-3356.

Cao, M., Du, P., Wang, X., Yu, Y.Q., Qiu, Y.H., Li, W., Gal-On, A., Zhou, C., Li, Y., and Ding, S.W. (2014). Virus infection triggers widespread silencing of host genes by a distinct class of endogenous siRNAs in Arabidopsis. Proc Natl Acad Sci USA 111, 14613-14618.

Chen, H.M., Chen, L.T., Patel, K., Li, Y.H., Baulcombe, D.C., and Wu, S.H. (2010). 22-Nucleotide RNAs trigger secondary siRNA biogenesis in plants. Proc Natl Acad Sci USA 107, 15269-15274.

Chen, X. (2005). microRNA biogenesis and function in plants. FEBS Lett 579, 5923-5931.

Chen, X. (2009). Small RNAs and their roles in plant development. Annu Rev Cell Dev Biol 25, 21-44.

Cogoni, C., and Macino, G. (2000). Post-transcriptional gene silencing across kingdoms. Curr Opin Genet Dev 10, 638-643.

Cogoni, C., Romano, N., and Macino, G. (1994). Suppression of gene expression by homologous transgenes. Antonie Van Leeuwenhoek 65 , 205-209

Creasey, K.M., Zhai, J., Borges, F., Van Ex, F., Regulski, M., Meyers,
B.C., and Martienssen, R.A. (2014). miRNAs trigger widespread epigenetically activated siRNAs from transposons in Arabidopsis. Nature 508, 411-415.

Cuperus, J.T., Carbonell, A., Fahlgren, N., Garcia-Ruiz, H., Burke, R.T., Takeda, A., Sullivan, C.M., Gilbert, S.D., Montgomery, T.A., and Carrington, J.C. (2010). Unique functionality of 22-nt miRNAs in triggering RDR6-dependent siRNA biogenesis from target transcripts in Arabidopsis. Nat Struct Mol Biol 17, 997-1003.

Fabian, M.R., Sonenberg, N., and Filipowicz, W. (2010). Regulation of mRNA translation and stability by microRNAs. Annu Rev Biochem 79, 351-379.

Fire, A., Xu, S., Montgomery, M.K., Kostas, S.A., Driver, S.E., and Mello, C.C. (1998). Potent and specific genetic interference by double-stranded RNA in Caenorhabditis elegans. Nature 391, 806-811.

Gasciolli, V., Mallory, A.C., Bartel, D.P., and Vaucheret, H. (2005). Partially redundant functions of Arabidopsis DICER-like enzymes and a role for DCL4 in producing trans-acting siRNAs. Curr Biol 15, 1494-1500.

Gazzani, S., Lawrenson, T., Woodward, C., Headon, D., and Sablowski, R. (2004). A link between mRNA turnover and RNA interference in Arabidopsis. Science 306, 1046-1048.

Ghildiyal, M., and Zamore, P.D. (2009). Small silencing RNAs: an expanding universe. Nat Rev Genet 10, 94-108.

Gregory, B.D., O’Malley, R.C., Lister, R., Urich, M.A., Tonti-Filippini, J., Chen, H., Millar, A.H., and Ecker, J.R. (2008). A link between RNA metabolism and silencing affecting Arabidopsis development. Dev Cell $14,854-866$.

Gy, I., Gasciolli, V., Lauressergues, D., Morel, J.B., Gombert, J., Proux, F., Proux, C., Vaucheret, H., and Mallory, A.C. (2007). Arabidopsis FIERY1, XRN2, and XRN3 are endogenous RNA silencing suppressors. Plant Cell 19, 3451-3461.

Halbach, F., Reichelt, P., Rode, M., and Conti, E. (2013). The yeast ski complex: crystal structure and RNA channeling to the exosome complex. Cell 154, 814-826.

Hamilton, A.J., and Baulcombe, D.C. (1999). A species of small antisense RNA in posttranscriptional gene silencing in plants. Science 286, 950-952.

Hutvagner, G. (2001). A cellular function for the RNA-interference enzyme Dicer in the maturation of the let- 7 small temporal RNA. Science 293, 834-838.

Incarbone, M., and Dunoyer, P. (2013). RNA silencing and its suppression: novel insights from in planta analyses. Trends Plant Sci 18, 382-392.

Johnson, A.W. (1997). Rat1p and Xrn1p are functionally interchangeable exoribonucleases that are restricted to and required in the nucleus and cytoplasm, respectively. Mol Cell Biol 17, 6122-6130.

Johnson, A.W., and Kolodner, R.D. (1995). Synthetic lethality of sep 1 (xrn1) ski2 and sepl (xrn1) ski3 mutants of Saccharomyces cerevisiae is independent of killer virus and suggests a general role for these genes in translation control. Mol Cell Biol 15, 2719-2727.

Kobayashi, K., Otegui, M.S., Krishnakumar, S., Mindrinos, M., and Zambryski, P. (2007). INCREASED SIZE EXCLUSION LIMIT2 encodes a putative DEVH box RNA helicase involved in plasmodesmata function during Arabidopsis embryogenesis. Plant Cell 19, 1885-1897.

Lam, P., Zhao, L.F., Eveleigh, N., Yu, Y., Chen, X.M., and Kunst, L. (2015). The exosome and trans-acting small interfering RNAs regulate cuticular wax biosynthesis during Arabidopsis inflorescence stem development. Plant Physiol 167, U323-U530.

Li, Y., Lu, J., Han, Y., Fan, X., and Ding, S. (2013). RNA interference functions as an antiviral immunity mechanism in mammals. Science $342,231-234$.

Lindbo, J.A., Silva-Rosales, L., Proebsting, W.M., and Dougherty, W.G. (1993). Induction of a highly specific antiviral state in transgenic plants: implications for regulation of gene expression and virus resistance. Plant Cell 5, 1749-1759.

Maillard, P.V., Ciaudo, C., Marchais, A., Li, Y., Jay, F., Ding, S.W., and Voinnet, O. (2013). Antiviral RNA interference in mammalian cells. Science 342, 235-238.

Marmont, A.M. (2000). New horizons in the treatment of autoimmune diseases: immunoablation and stem cell transplantation. Annu Rev Med 
$51,115-134$.

Martinez de Alba, A.E., Moreno, A.B., Gabriel, M., Mallory, A.C., Christ, A., Bounon, R., Balzergue, S., Aubourg, S., Gautheret, D., Crespi, M.D., Vaucheret, H., and Maizel, A. (2015). In plants, decapping prevents RDR6-dependent production of small interfering RNAs from endogenous mRNAs. Nucleic Acids Res 43, 2902-2913.

Montgomery, T.A., Howell, M.D., Cuperus, J.T., Li, D., Hansen, J.E., Alexander, A.L., Chapman, E.J., Fahlgren, N., Allen, E., and Carrington, J.C. (2008). Specificity of ARGONAUTE7-miR390 interaction and dual functionality in TAS3 trans-acting siRNA formation. Cell 133, $128-141$.

Moreno, A.B., Martinez de Alba, A.E., Bardou, F., Crespi, M.D., Vaucheret, H., Maizel, A., and Mallory, A.C. (2013). Cytoplasmic and nuclear quality control and turnover of single-stranded RNA modulate post-transcriptional gene silencing in plants. Nucleic Acids Res 41, 4699-4708.

Napoli, C., Lemieux, C., and Jorgensen, R. (1990). Introduction of a chimeric chalcone synthase gene into petunia results in reversible co-suppression of homologous genes in trans. Plant Cell 2, 279-289.

Olmedo, G., Guo, H., Gregory, B.D., Nourizadeh, S.D., Aguilar-Henonin, L., Li, H., An, F., Guzman, P., and Ecker, J.R. (2006). ETHYLENE-INSENSITIVE5 encodes a $5^{\prime}-->3^{\prime}$ exoribonuclease required for regulation of the EIN3-targeting F-box proteins EBF1/2. Proc Natl Acad Sci USA 103, 13286-13293.

Potuschak, T., Vansiri, A., Binder, B.M., Lechner, E., Vierstra, R.D., and Genschik, P. (2006). The exoribonuclease XRN4 is a component of the ethylene response pathway in Arabidopsis. Plant Cell 18, 3047-3057.

Pumplin, N., and Voinnet, O. (2013). RNA silencing suppression by plant pathogens: defence, counter-defence and counter-counter-defence. Nat Rev Microbiol 11, 745-760.

Ren, G., Xie, M., Zhang, S., Vinovskis, C., Chen, X., and Yu, B. (2014). Methylation protects microRNAs from an AGO1-associated activity that uridylates 5' RNA fragments generated by AGO1 cleavage. Proc Natl Acad Sci USA 111, 6365-6370.

Schubert, D., Lechtenberg, B., Forsbach, A., Gils, M., Bahadur, S., and Schmidt, R. (2004). Silencing in Arabidopsis T-DNA transformants: the predominant role of a gene-specific RNA sensing mechanism versus position effects. Plant Cell 16, 2561-2572.

Sijen, T., Fleenor, J., Simmer, F., Thijssen, K.L., Parrish, S., Timmons, L., Plasterk, R.H., and Fire, A. (2001). On the role of RNA amplification in dsRNA-triggered gene silencing. Cell 107, 465-476.

Sontheimer, E.J. (2005). Assembly and function of RNA silencing complexes. Nat Rev Mol Cell Biol 6, 127-138.

Thran, M., Link, K., and Sonnewald, U. (2012). The Arabidopsis DCP2 gene is required for proper mRNA turnover and prevents transgene silencing in Arabidopsis. Plant J 72, 368-377.
Vaistij, F.E., Jones, L., and Baulcombe, D.C. (2002). Spreading of RNA targeting and DNA methylation in RNA silencing requires transcription of the target gene and a putative RNA-dependent RNA polymerase. Plant Cell 14, 857-867.

Van der Krol, A.R., Mur, L.A., Beld, M., Mol, J.N., and Stuitje, A.R. (1990). Flavonoid genes in petunia: addition of a limited number of gene copies may lead to a suppression of gene expression. Plant Cell 2, 291-299.

Vaucheret, H., Beclin, C., Elmayan, T., Feuerbach, F., Godon, C., Morel, J.B., Mourrain, P., Palauqui, J.C., and Vernhettes, S. (1998). Transgene-induced gene silencing in plants. Plant J 16, 651-659.

Vazquez, F., Vaucheret, H., Rajagopalan, R., Lepers, C., Gasciolli, V., Mallory, A.C., Hilbert, J.L., Bartel, D.P., and Crete, P. (2004). Endogenous trans-acting siRNAs regulate the accumulation of Arabidopsis mRNAs. Mol Cell 16, 69-79.

Voinnet, O., Vain, P., Angell, S., and Baulcombe, D.C. (1998). Systemic spread of sequence-specific transgene RNA degradation in plants is initiated by localized introduction of ectopic promoterless DNA. Cell 95, 177-187.

Xia, R., Meyers, B.C., Liu, Z., Beers, E.P., Ye, S., and Liu, Z. (2013). MicroRNA superfamilies descended from miR390 and their roles in secondary small interfering RNA Biogenesis in Eudicots. Plant Cell 25, $1555-1572$.

Xia, R., Ye, S., Liu, Z., Meyers, B.C., and Liu, Z. (2015). Novel and recently evolved microRNA clusters regulate expansive $F$-BOX gene networks through phased small interfering RNAs in wild diploid strawberry. Plant Physiol 169, 594-610.

Xia, R., Zhu, H., An, Y.Q., Beers, E.P., and Liu, Z. (2012). Apple miRNAs and tasiRNAs with novel regulatory networks. Genome Biol 13, R47.

Xie, Z., Allen, E., Wilken, A., and Carrington, J.C. (2005). DICER-LIKE 4 functions in trans-acting small interfering RNA biogenesis and vegetative phase change in Arabidopsis thaliana. Proc Natl Acad Sci USA $102,12984-12989$.

Xie, Z., Johansen, L.K., Gustafson, A.M., Kasschau, K.D., Lellis, A.D., Zilberman, D., Jacobsen, S.E., and Carrington, J.C. (2004). Genetic and functional diversification of small RNA pathways in plants. PLoS Biol 2, e104.

Zhai, J., Jeong, D.H., De Paoli, E., Park, S., Rosen, B.D., Li, Y., González, A.J., Yan, Z., Kitto, S.L., Grusak, M.A., Jackson, S.A., Stacey, G., Cook, D.R., Green, P.J., Sherrier, D.J., and Meyers, B.C. (2011). MicroRNAs as master regulators of the plant NB-LRR defense gene family via the production of phased, trans-acting siRNAs. Genes Dev 25, 2540-2553.

Zhang, X., Zhu, Y., Liu, X., Hong, X., Xu, Y., Zhu, P., Shen, Y., Wu, H., Ji, Y., Wen, X., Zhang, C., Zhao, Q., Wang, Y., Lu, J., and Guo, H. (2015). Suppression of endogenous gene silencing by bidirectional cytoplasmic RNA decay in Arabidopsis. Science 348, 120-123.

Open Access This article is distributed under the terms of the Creative Commons Attribution License which permits any use, distribution, and reproduction in any medium, provided the original author(s) and source are credited. 